\title{
Measurement of near-wall stratified bubbly flows using electrical impedance
}

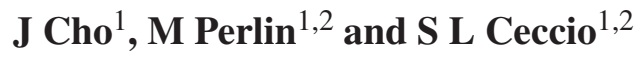 \\ ${ }^{1}$ Department of Mechanical Engineering, University of Michigan, Ann Arbor, MI 48109, \\ USA \\ ${ }^{2}$ Department of Naval Architecture and Marine Engineering, University of Michigan, \\ Ann Arbor, MI 48109, USA \\ E-mail: jinhyunc@engin.umich.edu,perlin@umich.edu and ceccio@umich.edu
}

Received 9 July 2004

Published 14 March 2005

Online at stacks.iop.org/MST/16/1021

\begin{abstract}
The impedance measurement between a pair of flush-mounted electrodes was used to measure the characteristics of a stratified near-wall bubbly flow. The variation in cross electrode impedance with liquid layer thickness and mixture void fraction was examined using numerical simulations and static experiments. The experimental realization of the measurement system was used to measure the solid fraction of a water-glass sphere mixture to an uncertainty of $\pm 2.4 \%$, where the diameter of the glass spheres ranged from 0.1 to 0.2 of the electrode diameter. A stratified bubbly flow was produced over a flat surface, and optical measurements of the bubble distributions were used to understand the measured impedances across the electrode pair. Comparison between the computed impedance change (based on the observed void fraction and liquid layer height) and the inferred quantities from the impedance measurement alone yielded a variation from 12 to $28 \%$ on average. The use of multiple electrode pairs is discussed.
\end{abstract}

Keywords: bubbly flows, void fraction, impedance measurements, microbubble drag reduction

(Some figures in this article are in colour only in the electronic version)

\section{Introduction}

Hydrodynamic drag reduction technology for high-speed vessels has been of great interest for at least the last four decades. Researchers have achieved as much as $80 \%$ skin friction drag reduction by injecting gas bubbles into the nearsurface boundary layer during laboratory experiments. The process of micro-bubble drag reduction has been explored by many previous researchers, and reviews of this topic are provided by Merkle and Deutsch [1] and Sanders et al [2].

A particular characteristic of near-wall bubbly shear flows associated with bubbly shear flows is the possibility of bubble stratification. Lift, drag and buoyancy forces on the bubbles can result in the migration of the bubbles from the wall and the formation of a near-single phase liquid layer [1, 25], shown schematically in figure 1 . The process of bubble migration and stratification in the near-wall region of a bubbly flow is critical to the study of bubble drag reduction, as bubble depletion near the wall will significantly reduce the effectiveness and persistence of the micro-bubble drag reduction effect.

Thus, a key to understanding microbubble drag reduction mechanisms is knowledge of the bubbly phase distribution near the solid surface. However, the presence of a high concentration of bubbles precludes many experimental diagnostics. In particular, optical access is limited by high bubble concentration so that many standard optical techniques, such as light scattering and laser Doppler velocimetry, are not useful due to multiple scattering of the light through the bubbly mixture. Photography is possible through transparent boundaries, but this method is often cumbersome and the images can be difficult to interpret.

Since bubbles and the surrounding liquid have contrasting electrical properties, it is possible to relate a measurement of bulk impedance to properties of the bubbly mixture, such as void fraction. Electrical impedance probes have been used for multiphase flow research since the late 1950s. The 


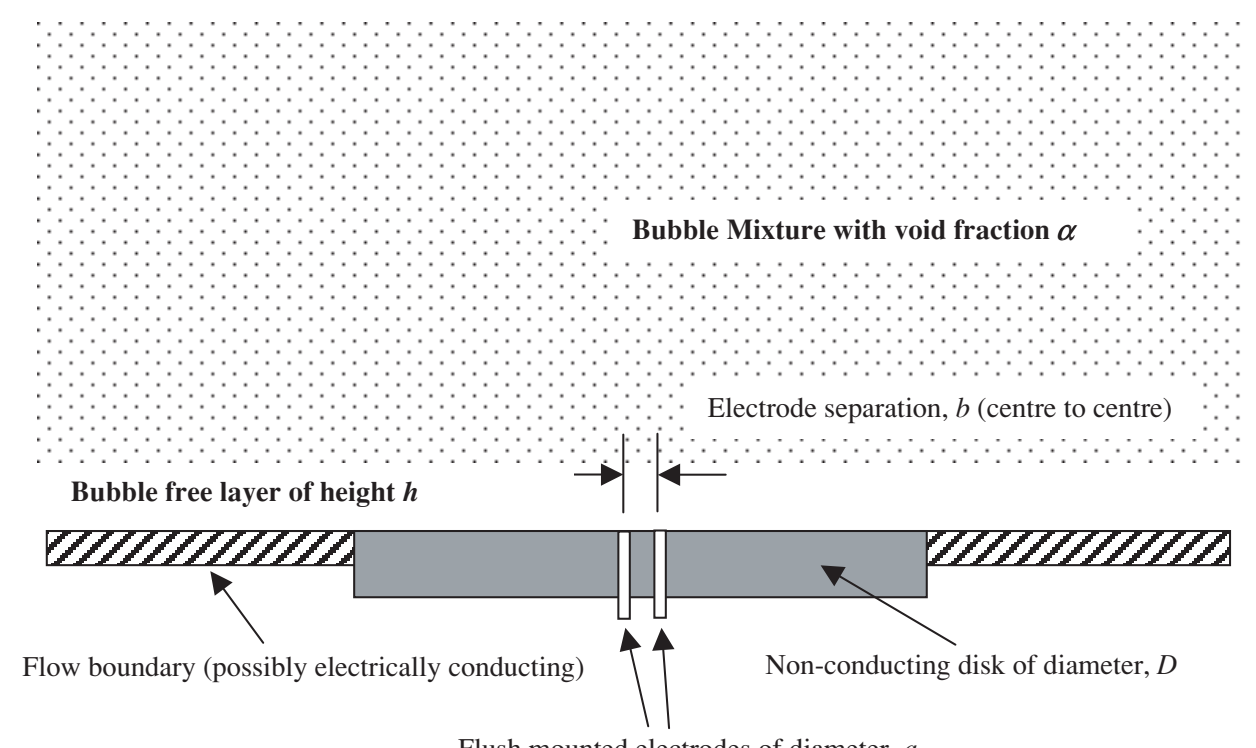

Flush mounted electrodes of diameter, $a$

Figure 1. A schematic diagram of the electrode configuration is shown. A pair of circular electrodes with diameter $a$ is separated by a centre-to-centre distance $b$. The electrodes are embedded in an electrically non-conducting disc with diameter $D$. A bubbly mixture with void fraction $\alpha$ is separated from the electrode plane by a single-phase liquid layer of thickness $h$.

theoretical basis for impedance techniques was established by Maxwell [3] who found that the void fraction can be obtained by measuring the impedance of the region when fine solid particles are uniformly dispersed in a continuum. Hewitt [4] suggested a detailed method for measuring the bulk void fraction relating the measured electrical property to the phase fraction using the classical Maxwell equation. A variety of impedance probes have been devised to measure bulk average void fraction [5-7], local void fraction [8-13] and film thickness [14-23]. Ceccio and George [24] provide a recent review. For near wall void fraction measurements, flush-mounted electrodes have been used successfully.

We wish to measure the void fraction of a bubbly flow over the flush mounted pair. The average void fraction (volume fraction) of the bubbly flow is $\alpha$, but there may exist a liquid layer of height $h$ above the flow boundary. If no liquid layer exists $(h=0)$, the bulk impedance between the electrodes can be related to the void fraction of the disperse phase in the traditional methods discussed in [24]. It is usually assumed that the average size of the disperse phase (such as the bubble diameter) is much smaller than the typical dimension of the electrode. Moreover, a mixture model is used to relate the bulk conductivity (and the known electrical conductivity of the continuous and disperse phases) with the volume fraction of the disperse phase [24, 26]. However, this methodology may fail if a significant region of bubble-free liquid exists near the surface.

In the present work, we wish to determine how the presence of the liquid layer will alter the conventional interpretation of the measured impedance. We will determine how sensitive the measurement of the void fraction is when a liquid layer is present. To do this, we will compute the relationship between the measured impedance and the characteristics of the bubbly flow using the simplest electrode geometry: a pair of flush mounted electrodes. We will then validate the computation with experimental measurements. Lastly, we will explain how multiple measurements can be used to measure both the void fraction and the layer height.

\section{Scaling the impedance across the electrode pair for stratified bubbly flow}

Figure 1 shows a schematic diagram of the flush mounted electrode pair. Two circular electrodes of diameter, $a$, are separated by a centre-to-centre distance $b$ on a planar boundary. An electrical potential is created across the electrodes, and the resulting current is measured. The conducting electrodes are mounted on a non-conducting substrate with a diameter $D$ much larger than $a$, and we can therefore assume that the 'leakage' electrical currents between the electrodes and grounded conducting surfaces far from the electrodes are negligible. Hence we assume that all of the current sourced from one electrode is sunk into the second. A layer of liquid with thickness, $h$, separates the plane of the electrodes from a layer of bubbly mixture with void fraction, $\alpha$.

The electrical impedance across the electrode pair, $Z_{P}$, is a function of geometric, physical and flow parameters and can be expressed as:

$$
Z_{P}=f_{1}\left(\alpha, d_{B}, h, a, b, \sigma_{C}, \sigma_{D}, Z_{P O}\right)
$$

where $\sigma_{C}$ and $\sigma_{D}$ are the electrical conductivity of the continuous phase (water) and the disperse phase (air), and $Z_{P O}$ is the impedance across the electrode pair when the domain is completely free of the disperse phase (i.e. without bubbles). We will assume that $\sigma_{C} \gg \sigma_{D}$, and thus $\sigma_{D} \approx 0$. Also, $d_{B}$ is the average bubble diameter, and we will assume that $a \gg d_{B}$, and thus $d_{B} \approx 0$. We can now produce two dimensionless groups

$$
\frac{Z_{P}-Z_{P O}}{Z_{P O}}=f_{2}\left(\alpha, \frac{h}{b}, \frac{a}{b}\right) .
$$

For the case of fixed electrode geometry, the change in measured impedance from the baseline configuration is related to the void fraction and the relative height of the liquid layer. 


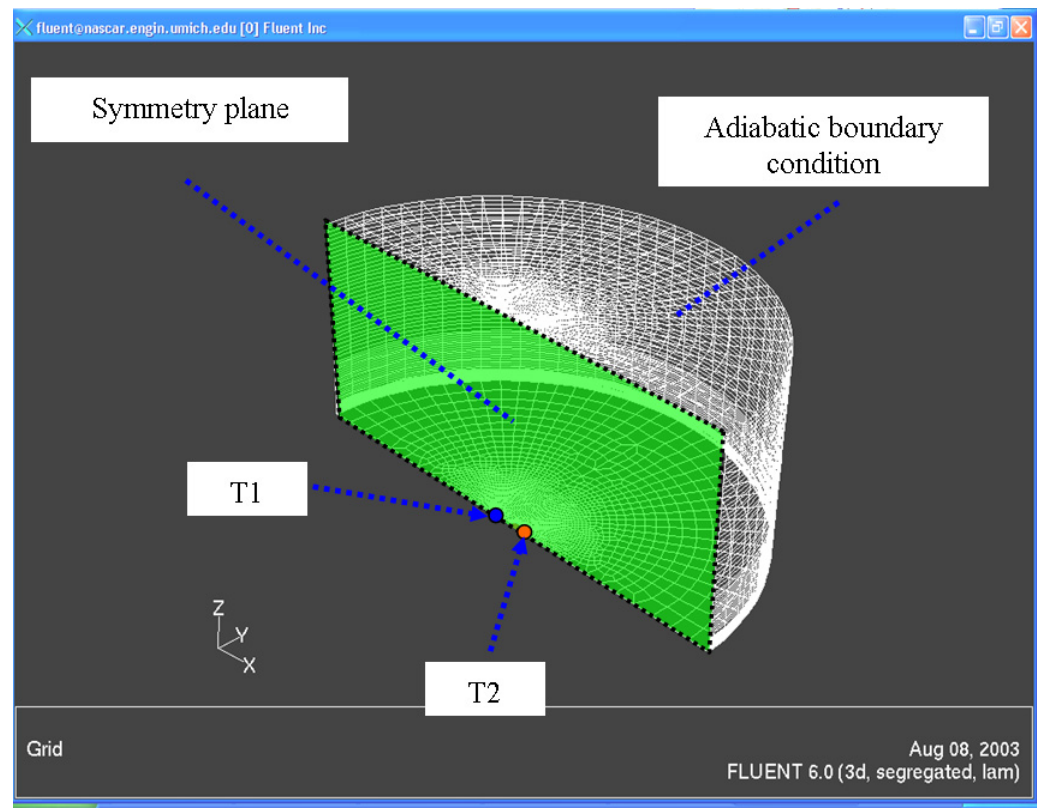

Figure 2. Mesh of the numerical model used to simulate the conduction between the electrode pair. The green area depicts the plane of symmetry while the white area shows where the adiabatic condition is applied.

\section{Computation of the impedance across the electrode pair}

Usually alternating voltages are used in the implementation of electrical impedance probes to avoid polarization effects at the electrodes. For ac electric conduction with field frequencies of the order of tens of megahertz or lower, the electric potential, $V$, within the conducting domain satisfies the Laplace equation

$$
\nabla \cdot \sigma \nabla V=0
$$

Therefore, the electric conduction field around the electrodes can be considered to satisfy the Laplace equation at the field frequency of $50 \mathrm{kHz}$ [27]. As with the electric conduction field created by the electrode pair, a temperature field with no heat generation also satisfies the Laplace equation. Mathematically, temperature is equivalent to electric potential while thermal conductivity is equivalent to electric conductance. Therefore, the electrical conduction between the electrode pair can be simulated through a solution of an analogous heat conduction problem. The commercial software package 'FLUENT' was used to conduct such a simulation. The particular values for $a(3.18 \mathrm{~mm}), D(114.3 \mathrm{~mm})$ and $b(6.35 \mathrm{~mm})$ were chosen to correspond to the experiments described below.

Figure 2 shows the mesh and boundary conditions used in the simulation. In the orthogonal coordinate system represented by $(x, y, z)$, the simulation domain is $(-50 \mathrm{~mm} \leqslant$ $x \leqslant 50 \mathrm{~mm}),\left(0 \mathrm{~mm} \leqslant y \leqslant \sqrt{50^{2}-x^{2}} \mathrm{~mm}\right),(0 \mathrm{~mm} \leqslant z \leqslant$ $53 \mathrm{~mm})$. Symmetry of the electrode pair allows the modelling of a half domain. A symmetry boundary condition is applied to the vertical mid-plane. Two semi-circles with radius $1.59 \mathrm{~mm}$ centred at $(3.18 \mathrm{~mm}, 0 \mathrm{~mm}, 0 \mathrm{~mm})$ and $(-3.18 \mathrm{~mm}$, $0 \mathrm{~mm}, 0 \mathrm{~mm}$ ) were used to represent the electrodes, and a distinct constant temperature was imposed on each respectively. On the domain exterior, an adiabatic condition was applied. The adiabatic condition for the exterior is based on the assumption that the domain is sufficiently large that there is no significant heat transfer beyond its boundary.

The spatial conductivity distribution of the domain can be prescribed through the assignment of the domain thermal conductivity. The conductivity of the bubble-laden portion of the domain was computed with the mixture relationship of Maxwell [3]. If the disperse phase can be considered a uniformly disperse mixture, the void fraction, $\alpha$, can be determined from the conductance of the continuous phase (water) and the conductance of the mixture by applying Maxwell's equation

$$
\alpha=\frac{2 \sigma_{C}+\sigma_{D}-2 \sigma_{M C}+\frac{\sigma_{M C} \sigma_{D}}{\sigma_{C}}}{\sigma_{M C}-\frac{\sigma_{D}}{\sigma_{C}}+2\left(\sigma_{C}-\sigma_{D}\right)} .
$$

In this formulation $\sigma_{M C}$ is the conductivity of the bubbly mixture. With $\sigma_{C} \gg \sigma_{D}$,

$$
\alpha=\frac{2 \sigma_{C}-2 \sigma_{M C}}{\sigma_{M C}+2 \sigma_{C}} .
$$

This relationship was used to prescribe the impedance of the bubbly mixture in the computational domain.

Simulation results of dimensionless impedance change as a function of void fraction and dimensionless mixture location with dimensionless $a / b=\frac{1}{2}$ are shown in figure 3 . The $e$-folding depth (i.e. $\mathrm{e}^{-\pi}$ decay) is found to be a depth of $\sim 0.5$. The cross-electrode impedance shows discernable changes with mixture void fraction when $h / b$ is less than about 0.3 , and it changes strongly when $h / b$ is less than about 0.1 .

\section{Measurement of the impedance across the electrode pair}

A series of experiments were conducted that accompanied the computations described above. Figure 4 shows an electrical schematic for the ac bridge that was used to measure the change in impedance across the electrode pair. The frequency range 


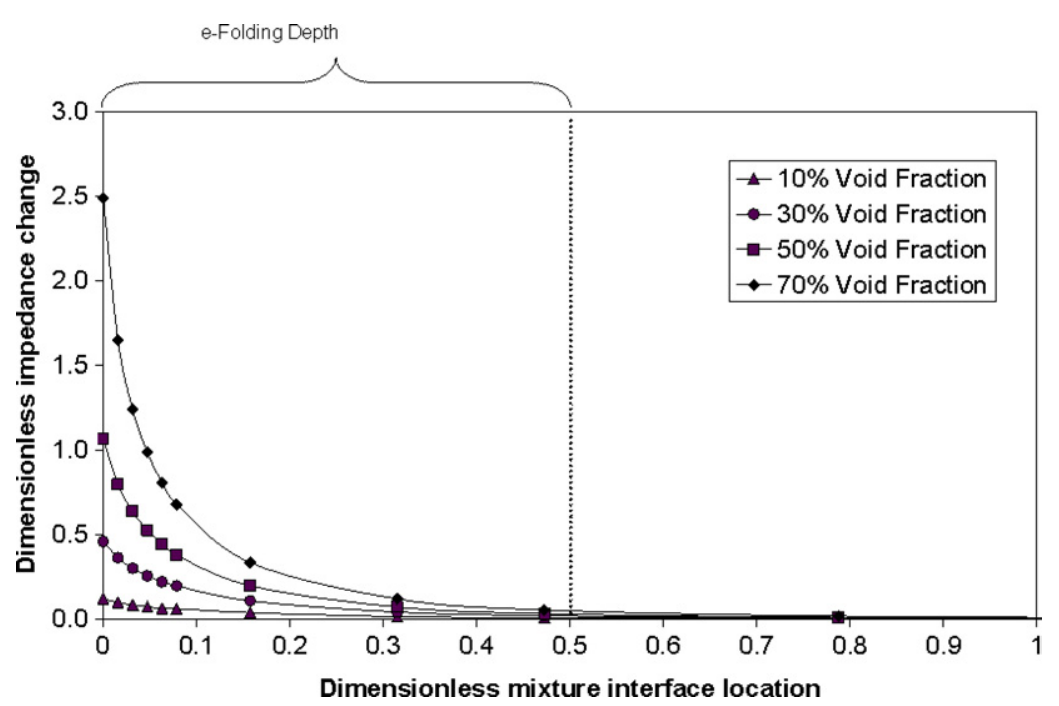

Figure 3. Simulation results of the dimensionless impedance change as a function of void fraction and dimensionless mixture interface location when the dimensionless radius $a / b=0.5$.

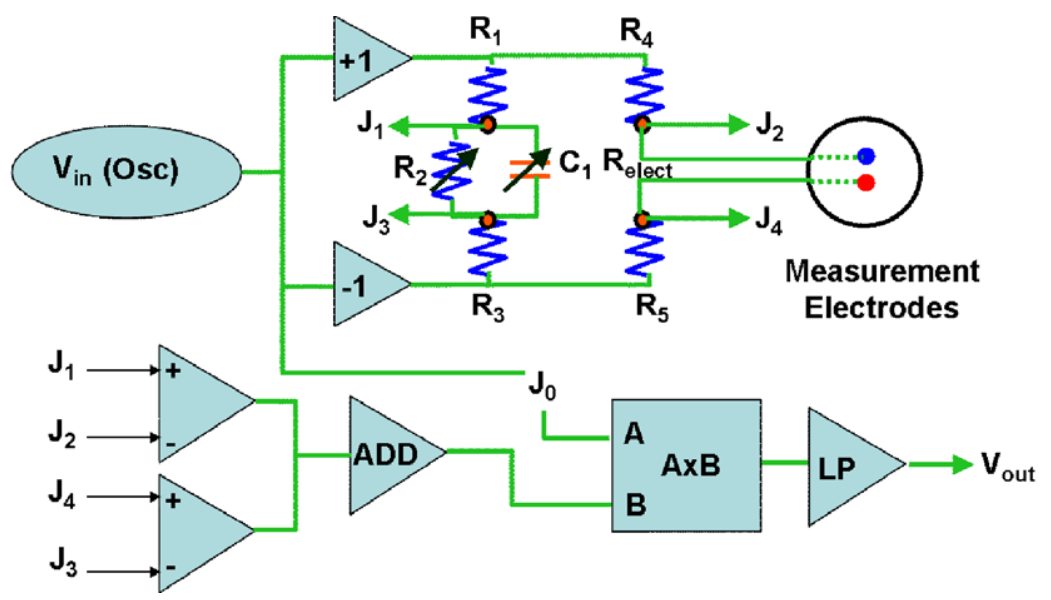

Figure 4. A block schematic diagram of the ac bridge and demodulator used in the experiments.

selected for the current determines which component of the total impedance dominates the measurement. As the applied frequency is increased, the capacitance of the phases will dominate the bulk admittance [27]. For air-water mixtures, the frequency of operation found in the literature ranges from $5 \mathrm{kHz}$ to $5 \mathrm{MHz}$. In the system used in the present study, the oscillator provides a $50 \mathrm{kHz}$ signal to excite the bridge. At $50 \mathrm{kHz}$, the resistance component of the phases dominates the impedance measurement.

The two electrodes are excited with voltages that are $180^{\circ}$ out-of-phase. The electrodes act as an element in the leg of an electrical bridge. In a first-order approximation, the electrode impedance across the electrodes can be modelled as a resistor and capacitor in parallel. (The capacitance is required primarily to null the impedance contribution of the cables leading to the electrode pair.) A variable resistor and variable capacitor are used to zero the bridge. If the impedance between the electrodes departs from the null condition, a difference voltage is produced at the bridge. This signal is then demodulated and low-pass filtered at $10 \mathrm{kHz}$. The bridge can be used to measure the absolute impedance across the pair, and it is sensitive to small impedance changes centred on this baseline value.

To assess the uncertainty associated with the electrical bridge, tests were performed using resistors of known resistance. A digital multimeter (Fluke 112) was employed to accurately measure the test resistors. The accuracy of the multimeter is $\pm 0.9 \%$. Resistors with a $5 \%$ error specification were used. Measured resistances using the multimeter were all within 5\% as expected. First, a base resistor was selected (a $15.91 \mathrm{k} \Omega$ resistor was used) and connected across the electrodes of the bridge. Next, the bridge was balanced, and the output voltage was measured at a $1 \mathrm{kHz}$ sampling rate for $3 \mathrm{~s}$. The variable resistor was changed incrementally six times to $5.0,10.0,15.0,20.0,25.0$ and $32.0 \mathrm{k} \Omega$ and the output voltages were recorded at each step. Then, the variable resistor was set to the value and again the bridge was balanced. A second-order polynomial was used to fit the calibration curve using the least-squares method. The calibration points had a standard deviation of approximately $0.4 \mathrm{k} \Omega$ relative to the fitted curve. Next, seven different resistors which spanned the calibration resistance range $(19.0,20.8,25.0,28.0,31.8,36.5$ 


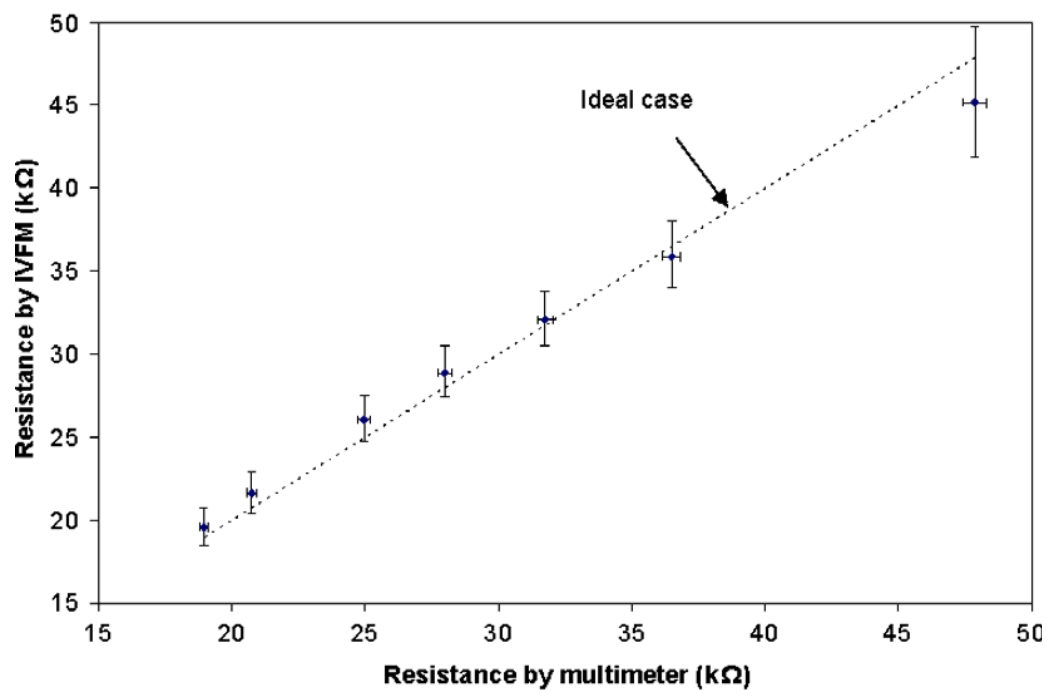

Figure 5. Comparison between the resistances measured by the multi-meter and calculated from the calibration curve fit. The dotted line represents the ideal case where the calculated resistance perfectly matches the measured resistance.

and $47.9 \mathrm{k} \Omega$ ) were selected for the test, and the resistance values were measured precisely. Each of the seven resistors was connected across the electrodes of the bridge, and the output voltages were recorded at a sampling rate of $1 \mathrm{kHz}$ for $3 \mathrm{~s}$. The average output voltages associated with the seven resistors were substituted into the fitted calibration curve to obtain the calibrated resistance output. Figure 5 shows the results. The standard deviations of the output signals were used to calculate the vertical error bars. The error associated with the multimeter resolution $(0.9 \%)$ was used for the horizontal error bars.

\section{Results for measurement under static conditions}

A pair of electrodes was fabricated with $a=3.18 \mathrm{~mm}, D=$ $114.3 \mathrm{~mm}$ and $b=6.35 \mathrm{~mm}$. The electrodes were made of polished brass, and the substrate was made of PVC. Two coaxial cables of length varying from 1 to $10 \mathrm{~m}$ were used to connect the electrode pair to the impedance bridge.

First, an electrode pair was placed in a water tank such that a liquid layer of known thickness separated it from a solid, non-conducting boundary, corresponding to the case of $\alpha=1$. In this experiment, a prescribed water layer thickness was achieved by placing spacers of known thickness between the electrode plate and the tank bottom. The bottom of the tank is constructed of cast acrylic. The electrical conductance of the cast acrylic is very small compared to that of water (i.e. water: $10^{2}-10^{3} \mu$ mho $\mathrm{cm}^{-1}$; acrylic: order of $10^{-10}$ $\mu \mathrm{mho} \mathrm{cm}^{-1}$ ). Therefore, the bottom of the tank serves as a near perfect insulator. The resistance needed to balance the bridge was recorded for ten different liquid thicknesses. The error associated with the thickness measurement was within $0.013 \mathrm{~mm}$.

Corresponding equivalent numerical simulations were conducted to determine the impedance of each configuration. The water layer was simulated by prescribing the thermal conductivity in the model. Thermal conductivity was converted from the electrical conductance of the water measured by a conductivity meter with a resolution of
$1 \mu \mathrm{mho} \mathrm{cm}^{-1}$. In the simulation, the temperature difference between the two electrodes divided by the overall heat transfer was used for the impedance calculation. Figure 6 shows the impedance results as a function of water layer thickness. Impedance from the simulation matches very well with the experimental results. The impedance bridge was calibrated and corrected for bias errors by placing a series of precision resistors across the electrodes. Very good agreement is found between the numerical and experimental results.

Next, we examined the case of a liquid layer when $\alpha<1$ and $h=0$. Since the electrical conductance of an air bubble is much smaller than that of an equivalent volume of water, the bubble can be considered as an electrical non-conductor. Hence we used glass spheres in place of bubbles for the static test. The electrical conductance of glass is also much smaller than the electrical conductance of water (i.e. $\sigma_{1}=10^{2}-10^{3} \mu$ mho $\mathrm{cm}^{-1}$ for the water used; $\sigma_{2}=10^{-10}-10^{-15} \mu \mathrm{mho} \mathrm{cm}^{-1}$ for the glass spheres), and thus glass spheres can substitute for air bubbles with regard to electrical conductance. The distribution of the glass-sphere diameters used in the experiment ranged between 300 and $600 \mu \mathrm{m}$. The glass-sphere diameter to electrode diameter (i.e. $3.18 \mathrm{~mm}$ ) ratio in the experiment ranges from about 0.1 to 0.2 .

The void fraction of the mixture was calculated by physically measuring the mixture volume. $150 \mathrm{ml}$ of water and $150 \mathrm{ml}$ of glass spheres were mixed in a cylinder. The uncertainty of the volume estimation is about $1 \mathrm{ml}$. The total volume of the mixture was $192 \mathrm{ml}$. From these values the solid fraction of the glass-water determined was $64 \%$ with an uncertainty of $\pm 0.8 \%$. The void fraction measured between the electrode pair was determined by measuring the impedance of the mixture and converting this value to void fraction using Maxwell's equation. The average void fraction derived from the electrical measurement was $61.6 \%$ and the standard deviation was $0.55 \%$.

These non-flowing tests demonstrate that the impedance bridge used to measure the cross-electrode impedance is satisfactory, and it also demonstrates that the Maxwell mixture model can be used to relate the impedance of the bubbly mixture to the void fraction. 


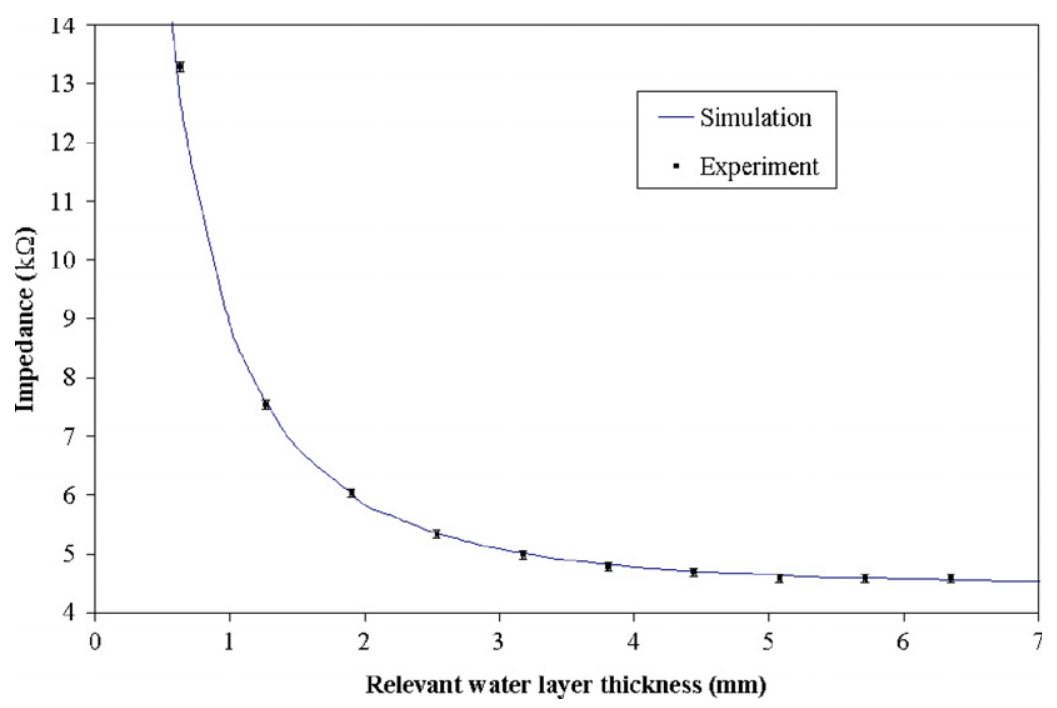

Figure 6. Comparison of the simulated impedance values and experimentally measured values for a water layer over the electrode pair bounded by a non-conducting interface $(h>0$ and $\alpha=1)$.

Output of the ac bridge
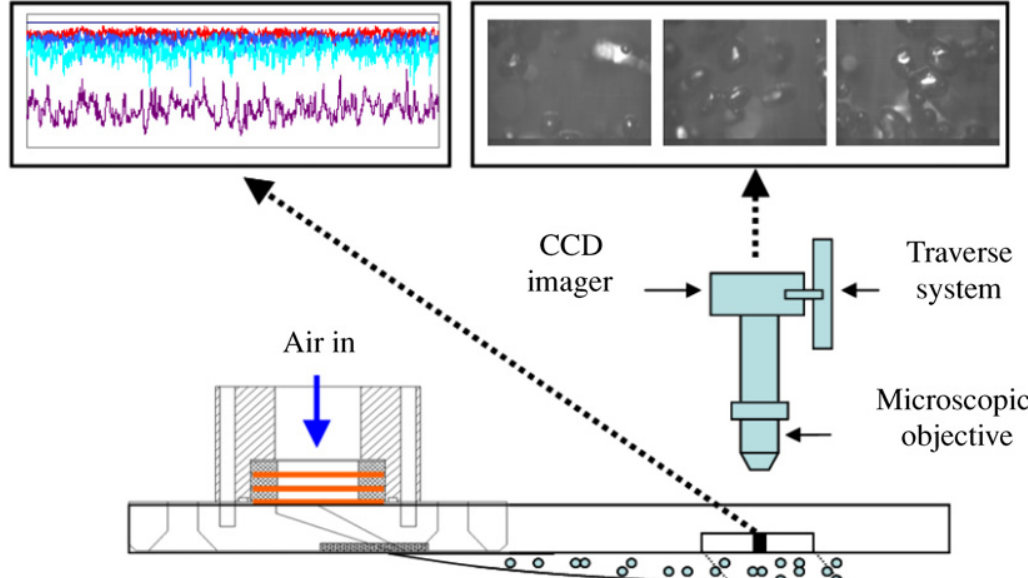

Freestream water
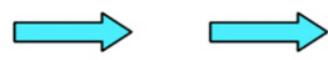

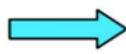

Electrodes

Bubble images
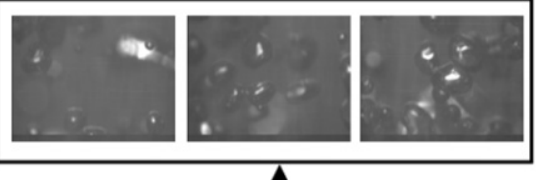

$\mathrm{CCD}$

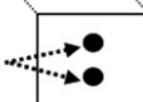

Tank wall

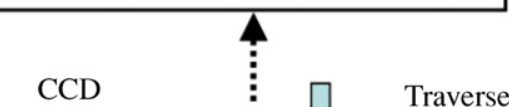

Figure 7. A schematic of the bubble injection experiment.

\section{Results from measurement of a bubbly shear flow}

We will now use the electrode pair to measure the impedance of a bubbly shear flow. Experiments were conducted in the University of Michigan's 9-Inch Cavitation Tunnel. The water tunnel has a circular contraction downstream of a series of flow management screens with contraction ratio 6.4:1. The test section has a $22.9 \mathrm{~cm}$ diameter round inlet that is faired to a rectangular test section with widely rounded corners. Four acrylic windows $(93.9 \mathrm{~cm} \times 10.0 \mathrm{~cm}$ viewing area) permit optical access to the test section flow. Figure 7 shows a schematic of the bubble injection experiment. To create the microbubble injected layer, compressed air was injected through a carefully designed injection system. The air was filtered, and then passed through screens with $0.5 \mathrm{~mm}$ diameter holes spaced $1 \mathrm{~mm}$ apart. The screens served to evenly distribute the airflow through a pressure drop. Prior to injection into the flow, the air passed through a $10^{\circ}$-contracting slot at a $25^{\circ}$ mean angle and a $1.35-\mathrm{mm}$-thick sintered metal plate with $40 \mu \mathrm{m}$ pores. The injector was located $10 \mathrm{~cm}$ downstream from the end of the contraction and an air flow meter with control valve was used for flow rate adjustment.

A pair of brass electrodes with the same electrode diameter and spacing used previously in the static experiments was flush mounted on the upper acrylic window surface located $33 \mathrm{~cm}$ downstream of the air injector slot. A digital camera system 
was used to inspect the bubbly boundary layer immediately adjacent to the surface of the plate. Images were captured using a Sony XC-55BB progressive scan black-white camera module. A C-mount adapter coupled with an extension ring produced a field of view (FOV) of $3.15 \mathrm{~mm} \times 2.25 \mathrm{~mm}$ (width $\times$ height). The uncertainty of the field of view was approximately $\pm 25 \mu \mathrm{m}$. The depth of field (DOF) was measured to be $0.67 \mathrm{~mm}$ and the uncertainty of the DOF was approximately $\pm 18 \mu \mathrm{m}$. A frame rate of $25 \mathrm{~Hz}$ and a $10 \mu \mathrm{s}$ shutter speed were used for all data acquisition. The entire imager setup was mounted to a traverse system that could be moved vertically with a micrometer controller.

Three nominal air injection rates, $240,320,400 \mathrm{~cm}^{3} \mathrm{~s}^{-1}$ at $10 \mathrm{~m} \mathrm{~s}^{-1}$ freestream speed were chosen for the test conditions in this experiment. The output signal of the ac bridge was recorded for $5 \mathrm{~s}$ at a sampling rate of $10 \mathrm{kHz}$. Careful consideration was used in selecting the focal planes in relation to the depth of field and the refraction index of water. The focal plane in water is changed by the refraction index times the distance the camera system is traversed in air. Taking the depth of field of the system as $670 \mu \mathrm{m}$ and the refractive index of water as 1.33, two appropriate distances of the traverse system were determined to be $250 \mu \mathrm{m}$ and $750 \mu \mathrm{m}$. When the traverse system was set to $250 \mu \mathrm{m}$, air bubbles that were present over the range of $0-670 \mu \mathrm{m}$ from the surface are focused. Likewise, bubbles within the range of $670 \mu \mathrm{m}$ to $1340 \mu \mathrm{m}$ are focused by setting the traverse system at $750 \mu \mathrm{m}$.

In processing the microbubble images the major task was to determine the area fraction of bubbles near the wall. In a multiphase flow, the fraction of bubble volume to total volume (the void fraction) is determined by calculating the ratio of air volume divided by the total volume of the air-water mixture. Usually the overall void fraction is calculated as the airflow rate divided by the combined flow rate of the air and water. However, since the electrode pair is very sensitive to the void fraction close to the wall, it is very important to analyse the bubble density directly at the surface, since the overall void fraction is not meaningful. From two-dimensional images the true volume density of air cannot be obtained. Instead a related quantity, the area ratio, was extracted from the images and serves as an alternative to the void fraction. This value represents the direct effect of the presence of the bubbles in the boundary layer near the wall. However, the area ratio is an over-prediction of the void fraction. If the bubble is a perfect sphere and bubble overlap is not a problem, the apparent void fraction of a bubble is about $2 / 3$ of the area ratio. The ratio of the void fraction to area ratio changes as the bubble distorts. The shapes of the bubbles in this test were not perfect spheres; thus, the void fraction is presumed to be larger than $2 / 3$ of the area ratio.

To determine the area ratios, the images were processed using Adobe Photoshop 7.0. The bubbles that were deemed 'in focus' were painted white while the background was painted black. A focused bubble was one that exhibited a distinct circumference. Figure 8 is a sample image at a $400 \mathrm{~cm}^{3} \mathrm{~s}^{-1}$ air flow rate. A MATLAB code was used to calculate the area ratio (painted bubble area/total picture area) for each image. Table 1 presents the results from this analysis.

A calculation of the expected impedance change was conducted based on the observed void fraction and liquid layer

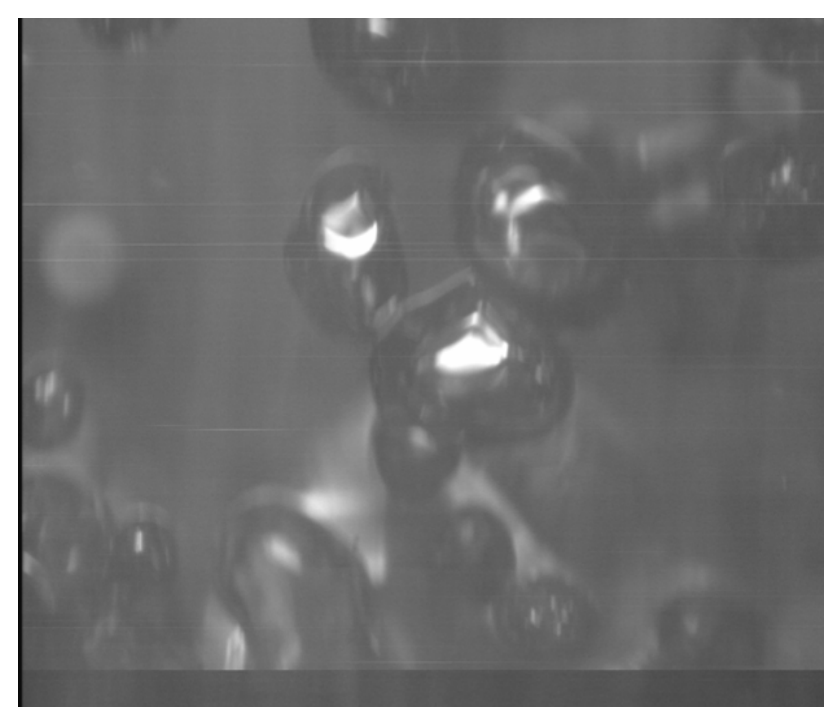

(a)

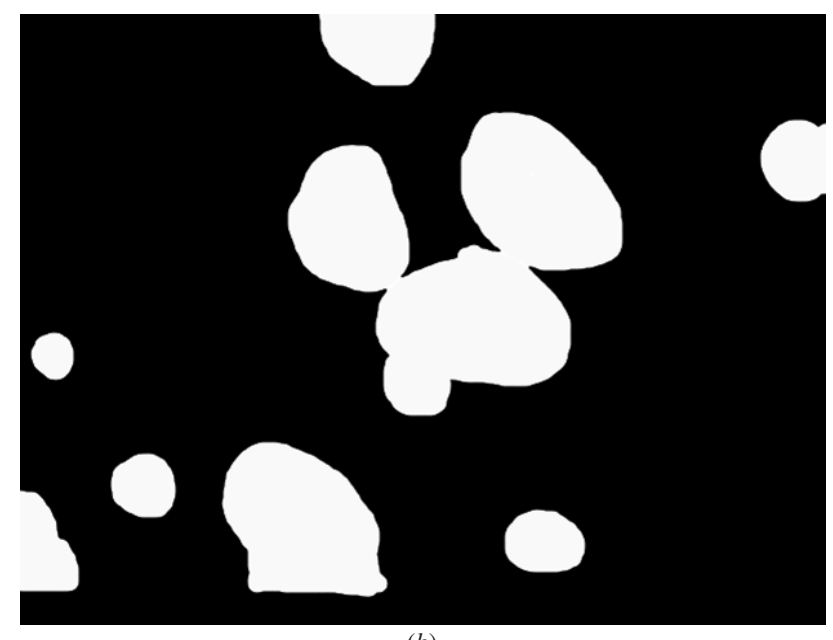

(b)

Figure 8. (a) Typical bubble image for $4.0 \times 10^{-4} \mathrm{~m}^{3} \mathrm{~s}^{-1}$ air injection rate at $10 \mathrm{~ms}^{-1}$ freestream velocity. (b) Processed image.

Table 1. Area ratio result for each air injection rate.

\begin{tabular}{lllll}
\hline & $\begin{array}{l}\text { Flow rate } \\
\left(\mathrm{cm}^{3} \mathrm{~s}^{-1}\right)\end{array}$ & $\begin{array}{l}\text { Free stream } \\
\text { velocity }\left(\mathrm{m} \mathrm{s}^{-1}\right)\end{array}$ & $\begin{array}{l}\text { Area ratio } \\
(0-670 \mu \mathrm{m})\end{array}$ & $\begin{array}{l}\text { Area ratio } \\
(670-1340 \mu \mathrm{m})\end{array}$ \\
\hline Case 1 & 240 & 10 & 0.036 & 0.128 \\
Case 2 & 320 & 10 & 0.027 & 0.152 \\
Case 3 & 400 & 10 & 0.027 & 0.230 \\
\hline
\end{tabular}

depth. The void fraction was assumed to be equal to the area ratio, and the Maxwell equation was evaluated to calculate the conductivity of each of the two layers. As shown in table 1 , the area ratio in the range of 670-1340 $\mu \mathrm{m}$ increases as the air injection rate increases. However, in the $0-670 \mu \mathrm{m}$ range, where sensitivity is much higher, the area ratio does not change much as the air flow rate increases. As the air flow rate rises, the impedance change also increases. However, it is not quite proportional because the area ratio within the highest sensitivity range is nearly unaffected by the air injection rate.

The impedance changes of the model and the experiment are shown in table 2. Uncertainty estimates of the simulation impedance changes were calculated using the uncertainty of 
Table 2. Comparison of the impedance change between calculation and experiment: (a) is the result assuming the area ratio as the void fraction and (b) is the result using the void fraction from the assumption that bubbles are perfect spheres.

\begin{tabular}{llllll}
\hline & $\begin{array}{l}\text { Simulation } \\
\text { impedance } \\
\text { change }(\Omega)(\mathrm{a})\end{array}$ & $\begin{array}{l}\text { Simulation } \\
\text { impedance } \\
\text { change }(\Omega)(\mathrm{b})\end{array}$ & $\begin{array}{l}\text { Experimental } \\
\text { impedance } \\
\text { change }(\Omega)\end{array}$ & $\begin{array}{l}\text { Difference } \\
(\%)(\mathrm{a})\end{array}$ & $\begin{array}{l}\text { Difference } \\
(\%)(\mathrm{b})\end{array}$ \\
\hline Case 1 & $639( \pm 1)$ & $407( \pm 1)$ & 527 & 21.3 & -22.8 \\
Case 2 & $697( \pm 1)$ & $457( \pm 1)$ & 676 & 3.1 & -32.5 \\
Case 3 & $953( \pm 2)$ & $599( \pm 1)$ & 862 & 10.5 & -30.6 \\
\hline
\end{tabular}

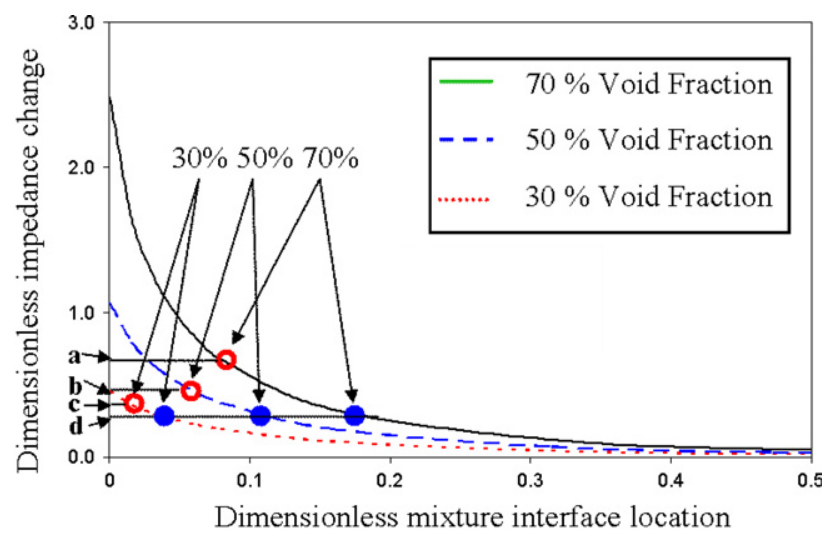

Figure 9. Illustration of the dimensionless impedance change as a function of the void fraction for two electrode pairs with separations $b$ and $2 b$. The dimensionless impedance change of the first pair is $\mathbf{d}$ (solid circles) and the impedance change for the second pair of electrodes can be $\mathbf{a}, \mathbf{b}$ or $\mathbf{c}$ (empty circles).

the DOF $( \pm 18 \mu \mathrm{m})$. Simulations were performed twice, once using the area ratio as the void fraction, once using the void fraction from the spherical bubble assumption. The simulations, using the area ratio as the void fraction, predicted a maximum $21.3 \%$ and an average $11.6 \%$ larger impedance change than did experiments. Using the void fraction with the bubbles assumed perfect spheres the simulation predicted a $28.6 \%$ smaller impedance change than did the experiments, on average. Bubbles were not extremely small spheres in this experiment. Therefore, the accuracy of the simulation for the dynamic bubble injection case is presumed to be between these two bounding limits.

\section{Estimation of the void fraction profile using multiple electrode pairs}

Two pairs of electrodes with different centreline distances and the same dimensionless diameters can be employed to estimate the mixture interface location and the void fraction near the surface. Consider two electrode pairs with separations $b$ and $2 b$. Figure 9 illustrates a posited condition where the dimensionless impedance change of the first pair of electrodes is assumed to be $\mathbf{d}$. There are infinite combinations of void fraction and dimensionless mixture interface location that would produce $\mathbf{d}$ for the first pair. That is, the solution to the inverse problem is not unique. However, the dimensionless impedance change experienced by the second pair of electrodes will be different for every combination. Three void fractions (i.e. $70 \%, 50 \%$ and $30 \%$ ) are selected for display. The dimensionless mixture location experienced by the second pair is half that experienced by the first pair.
Therefore, the impedance change of the second pair for each void fraction can be determined from the curves and is denoted as a, b, c. It is clear from figure 9 that the dimensionless impedance change of the second pair becomes larger as the void fraction increases. Therefore, a unique void fraction and mixture interface location that matches the impedance change of both the pairs can be determined.

Although any distance ratio between the two electrode pairs can be employed, there is a trade-off. For $h>0.3 b$, the electrodes are insensitive to the presence of the bubbly mixture. Consequently, some a priori information must be known about the liquid layer dimension to initially size the electrode spacing. Or, a series of electrode pairs with varying spacing (and diameters) can be employed. Multiple measurements of the cross-electrode impedances can then be used to find the best location of the mixture interface.

\section{Concluding remarks}

Impedance measurements between a pair of flush mounted electrodes were developed and employed to investigate the stratified bubbly flows near a solid surface. To interpret the impedance measurement, numerical simulations and static experiments were performed. The simulation model was validated through comparison with both static and dynamic experiments in a controlled environment. These experiments demonstrate that impedance changes between the electrodes can provide a measure of the liquid layer thickness beneath a bubbly mixture.

At least two electrode pairs must be used to uniquely determine the thickness of the liquid layer and the void fraction of the bubbly mixture, although some a priori knowledge about the dimension of the layer is needed to set the electrode geometry. Measurements across many electrode pairs could yield a more detailed gas phase distribution profile. Indeed, it is possible to use multiple electrodes and many combinations of impedance measurements to reconstruct more complex impedance distributions in a process known as electrical impedance tomography [28, 29]. The method described here may be considered a simplification of this general method.

\section{Acknowledgments}

This research was sponsored by the Defense Advance Research Projects Agency (Dr Lisa Porter, Program Manager) and the Office of Naval Research (Dr L Patrick Purtell, Program Manager) under ONR contract number N0001401-1-0880 and DARPA contract number HR0011-04-1-001. The content of this document does not necessarily reflect the position or the policy of the United States Government, and no official endorsement should be inferred. 


\section{References}

[1] Merkle C L and Deutsch S 1992 Drag reduction in liquid boundary layers by gas injection Prog. Astronaut. Aeronaut. $123351-412$

[2] Sanders W C, Dowling D R, Perlin M and Ceccio S L 2004 Bubble friction drag reduction in a high Reynolds number flat plate turbulent boundary layer J. Fluid Mech. submitted

[3] Maxwell J 1881 A Treatise on Electricity and Magnetism (Oxford: Clarendon)

[4] Hewitt G F 1978 Measurement of Two Phase Flow Parameters (London: Academic) pp 57-60, 182

[5] Lemonnier H, Nakach R, Favreau C and Selmer-Olsen S 1991 Sensitivity analysis of an impedance void meter to the void distribution in annular flow: a theoretical study Nucl. Eng. Des. 126 105-12

[6] Abouelwafa M S A and Kendall E J M 1980 The use of capacitance sensors for phase percentage determination in multiphase pipelines IEEE Trans. Instrum. 29 24-7

[7] Merilo M, Dechene R L and Cichowlas W M 1977 Void fraction measurement with a rotating electric field conductance gauge ASME J. Heat Transfer 99 330-2

[8] Ceccio S L and Brennen C E 1991 Observations of the dynamics and acoustics of traveling bubble cavitation $J$. Fluid Mech. 233 633-60

[9] Ceccio S L and Brennen C E 1992 Dynamics of attached cavities on bodies of revolution J. Fluids Eng. 114 93-9

[10] Calderbank P H, Pereira J and Burgess J M 1976 The physical and mass transfer properties of bubbles in fluidized beds of electrically conducting particles Fluidization Technology vol 1 ed D L Keairns (Washington, DC: Hemisphere) 115-67

[11] Gunn D J and Al-Doori H H 1985 The measurement of bubble flows in fluidized beds by electrical probes Int. J. Multiphase Flow 11 535-51

[12] van der Welle R 1985 Void fraction, bubble velocity and bubble size in two-phase flow Int. J. Multiphase Flow 11 317-45

[13] Clark N N, Seiss R and Turton R 1992 Probe measurements in gas-liquid systems Flow Meas. Instrum. 3 17-23

[14] Kim M and Kang H 1990 The development of a flush-wire probe and calibration technique for measuring liquid film thickness Advances in Gas Liquid Flows ed J H Kim, U S Rohatgi and A Hashemi (Dallas, TX: ASME) pp 131-8

[15] Cheremisinoff N P 1977 An experimental and theoretical investigation of horizontal stratified and annular two-phase flow with heat transfer $P h D$ Thesis Clarkson College of Technology Potsdam, NY
[16] Miya M, Woodmansee D E and Hanratty T J 1971 A model for roll waves in gas-liquid flow Chem. Eng. Sci. 26 1915-31

[17] Brown R C, Andreussi P and Zanelli S 1978 The use of wire probes for the measurement of liquid film thickness in annular gas-liquid flows Can. J. Chem. Eng. 56 754-7

[18] Lin P Y and Hanratty T J 1987 Detection of slug flow from pressure measurement Int. J. Multiphase Flow 13 $13-21$

[19] Koskie J E, Mudawar I and Tiederman W G 1989 Parallel-wire probes for the measurement of thick liquid films Int. J. Multiphase Flow 15 521-30

[20] Ruder Z, Hanratty P J and Hanratty T J 1989 Necessary condition for the existence of stable slugs Int. J. Multiphase Flow 15 209-26

[21] Özgü M R, Chen J C and Eberhardt N 1973 A capacitance method for measurement of film thickness in two-phase flow Rev. Sci. Instrum. 44 1714-6

[22] Delil A A M 1987 Feasibility demonstration of a sensor for high-quality two-phase flow Report No. NLR TR 87009 National Aerospace Laboratory, Amsterdam, The Netherlands pp 8-13

[23] Keska J K 1990 Some methodical and experimental aspects of measurement of a void fraction in gas-liquid flows Advances in Gas Liquid Flows ed J H Kim, U S Rohatgi and A Hashemi (Dallas, TX: ASME) pp 139-45

[24] Ceccio S L and George D L 1996 A review of electrical impedance techniques for the measurement of multiphase flows J. Fluids Eng. 118 391-9

[25] Pal S, Merkle C L and Deutsch S 1988 Bubble characteristics and trajectories in a microbubble boundary layer Phys. Fluids 31 744-51

[26] Louge M and Opie M 1990 Measurements of the effective dielectric permittivity of suspensions Powder Technol. 62 85-94

[27] Olsen H O 1967 Theoretical and experimental investigations of impedance void meters Report No 118 Institutt for Atomenergi, Kjeller Research Establishment, Kjeller, Norway

[28] Williams R A and Beck M S 1995 Process Tomography: Principles, Techniques, and Applications (Oxford: Butterworth-Heinemann)

[29] George D L, Torczynski J R, Shollenberger K A, O’Hern T J and Ceccio S L 2000 Validation of electrical impedance tomography for measurement of material distribution in two-phase flows Int. J. Multiphase Flow 26 549-81 